\section{Research Square}

Preprints are preliminary reports that have not undergone peer review.

They should not be considered conclusive, used to inform clinical practice, or referenced by the media as validated information.

\title{
Development and External Validation of a Nomogram for Early Predicting in- hospital Mortality of Patients with SARS-CoV-2 Pneumonia: A Two-Center, Retrospective Analysis
}

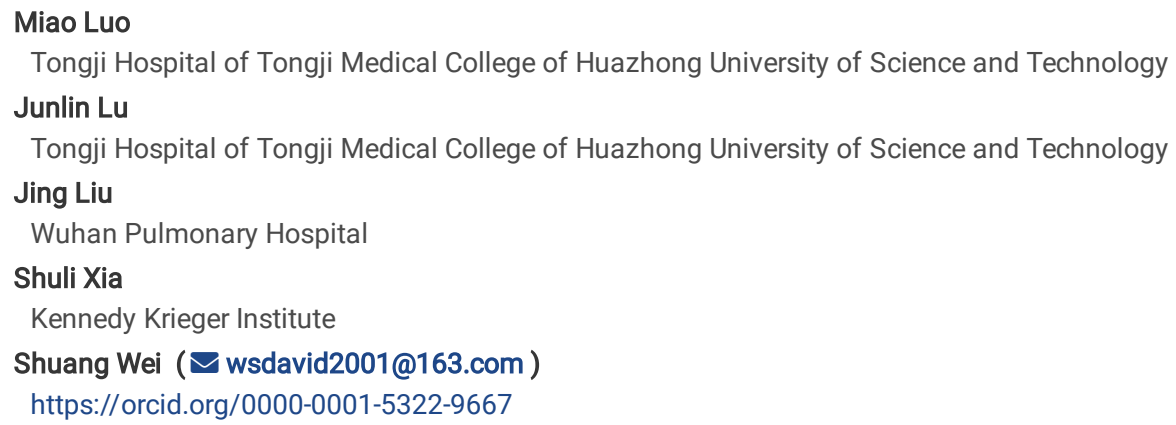

Research article

Keywords: SARS-CoV-2 pneumonia, nomogram, mortality

Posted Date: May 20th, 2020

DOI: https://doi.org/10.21203/rs.3.rs-27780/v1

License: (c) (1) This work is licensed under a Creative Commons Attribution 4.0 International License. Read Full License 


\section{Abstract}

Background At present, the death cases with SARS-CoV-2 pneumonia are continuing to increase globally. However, the information on death cases and predictive methods are substantial lacking. We aimed to develop a nomogram, which was validated by both internal and external cohorts, for early predicting mortality in hospitalized patients with SARS-CoV-2 pneumonia.

Methods We retrospectively collected data on 1,540 patients confirmed SARS-CoV-2 pneumonia from two hospitals. Multivariate logistic regression analysis was performed to examine factors associated with in-hospital mortality. We investigated the mortality related risk factors and their weights, thereafter designed and validated a predictive nomogram model to facilitate early discrimination of in-hospital death. We assessed the nomogram performance by examining calibration (calibration plots and Hosmer-Lemeshow calibration test) and discrimination (AUROC). We also plotted survival curves and decision curves to evaluate the clinical usefulness of the nomogram.

Results In the 1,540 patients from two centers, 248 cases died (16.1\%). In the predictive nomogram calculated by a multivariate logistic regression analysis, eight independent risk factors associated mortality included age $\geq 60$ years (odd ratio(OR) $=2.840 ; 95 \% \mathrm{Cl}, 1.467-5.495 ; P=0.002$ ), respiratory rate $\geq 30$ breaths per minute $(\mathrm{OR}=3.308 ; 95 \% \mathrm{Cl}, 1.408-7.770 ; P=0.006)$, neutrophil count $\geq 7 \times 10^{9} / \mathrm{L}(\mathrm{OR}=3.084 ; 95 \% \mathrm{Cl}, 1.667-5.707 ; P<0.001)$, lymphocyte count $\leq 0.8 \times 10^{9} / \mathrm{L}(\mathrm{OR}=4.688 ; 95 \% \mathrm{Cl}, 2.500-8.791 ; P<0.001)$, d-dimer $\geq 1.5 \mu \mathrm{g} / \mathrm{mL}(\mathrm{OR}=2.159 ; 95 \% \mathrm{Cl}, 1.169-3.989 ; P=0.014)$, lactate dehydrogenase $\geq$ $350 \mathrm{U} / \mathrm{L}(\mathrm{OR}=4.385 ; 95 \% \mathrm{Cl}, 2.299-8.362 ; P<0.001)$, procalcitonin $\geq 0.1 \mathrm{ng} / \mathrm{mL}(\mathrm{OR}=4.972 ; 95 \% \mathrm{Cl}, 2.537-9.746 ; P<0.001)$, and presence of myocardial injury $(\mathrm{OR}=2.289 ; 95 \% \mathrm{Cl}, 1.260-4.160 ; P=0.007)$ on admission. Calibration curves showed good fitting of the nomogram model with no statistical significance $(P$ $=0.740$ ) by Hosmer-Lemeshow test. This predictive nomogram had better predictive ability than CURB-65 score in training set (AUROC $=0.956$ vs $0.828, P<$ 0.001). The good predictive performance of the nomogram is suggested by calibration, discrimination, and survival curve analysis, whether in the training, internal or external validation set. The decision curve analysis showed that predicting mortality risk applying this nomogram would be better than having all patients or none patients.

Conclusions This nomogram is a reliable prognostic method that can accurately and early predict in-hospital mortality in patients with SARS-CoV-2 pneumonia. It can guide clinicians to improve their abilities to evaluate patient prognosis, enhance patient stratification, make earlier and reasonable decisions.

Trail registration: This is a retrospective observational study without a trial registration number.

\section{Introduction}

At the end of 2019, a strongly contagious viral pneumonia disease largely outbroke in Wuhan, Hubei province, China.[1] Within just three months, the new infection has spread throughout the country[2] and even global.[3, 4] A novel coronavirus named severe acute respiratory syndrome-coronavirus-2 (SARS-CoV2) was discovered and isolated from the bronchoalveolar lavage fluid of patients with novel pneumonia.[5, 6]

Until April 20, 2020, a total of 2,319,066 patients were diagnosed with SARS-Cov-2 pneumonia and 157,990 cases died globally. The current mortality rate is around $6.8 \%$. Presently most of the patients diagnosed with SARS-Cov-2 pneumonia are mild. Early clinical treatments have achieved gratifying results. Although the overall mortality rate is far lower than other members of coronaviridae, such as well-known severe acute respiratory syndrome coronavirus (SARS-CoV) and Middle East respiratory syndrome coronavirus (MERS-CoV),[7, 8] once the patients were diagnosed as critically illness, their risk of death increased sharply. A recent observational study showed that in a cohort of 52 patients with critical SARS-CoV-2 pneumonia, 32 (61.5\%) patients died in 28 days.[9]This mortality rate was much higher than severe SARS in Toronto (34\%)[10], Singapore (37\%)[11], and Hong Kong (26\%)[12] reported in the same period. Therefore, early discrimination of individuals at high risk of death is imperative for the patients with SARS-CoV-2 pneumonia. Our current study sought to uncover the clinical characteristics of non-survivors, develop and validate a nomogram that can predict mortality of SARS-CoV-2 pneumonia in an early stage.

\section{Methods}

\section{Study Design and Participants}

This two-center, retrospective study was done at Wuhan Pulmonary Hospital and Tongji Hospital affiliated to Huazhong University of Science and Technology in Wuhan, which are both designated hospitals to treat patients with SARS-CoV-2 pneumonia. We retrospectively analyzed 1,540 patients who were discharged or died from Jan 9, 2020 to March 24, 2020. All patients were diagnosed with SARS-CoV-2 pneumonia according to WHO interim guidance. The Ethics Commission of Tongji Hospital and Wuhan Pulmonary Hospital have all approved this study.

\section{Data Collection}

The data were collected from the hospital's electronic medical record system, which included demographic information, symptoms, comorbidities, complications, routine laboratory tests, immunological tests, computed tomography (CT) results, and clinical interventions. In order to early predict mortality, the data of the laboratory examinations and computed tomography (CT) findings on admission or in the first time during hospitalization was collected for analysis. CURB-65 score of each patient was calculated. The entry and calculation of all relevant data were verified by two experienced clinical researchers. The clinical outcomes of the patients included in this clinical study were observed until March 24, 2020. The outcome of this study was all-cause in-hospital mortality. 


\section{Statistical Analysis}

All patients were divided into two groups: survival and non-survival group. The comparisons were performed between those two groups. Normally distributed continuous variables were tested using t-test and characterized by mean \pm SD, while non-normally distributed continuous variables were compared by MannWhitney $\mathrm{U}$ test and characterized by median (interquartile range, IQR). The $\chi 2$ or Fisher exact tests were utilized for categorical variable analysis.

The percentages of missing values of variables in our cohort were lower than 50\%. Multiple imputation (MI) method was used to impute missing data in our cohort with the guidance of previous study[13]. Conclusions of univariate logistic regression analyses with or without imputed data were unchanged.

Continuous variables were categorized and retained for multivariate testing. Data of 1068 patients form Tongji Hospital was partitioned randomly into two complementary subsets: the training set of 749 (70\%) was used to establish the model; the testing set of 319 (30\%) was used for internal validation. Data of 472 patients from Wuhan Pulmonary Hospital was employed to validate the model externally. For mortality predictive model establishment, continuous variables were categorized by a cutoff point. The cutoff value was confirmed from Youden's index of receiver operator characteristic (ROC) curve. Variables with $P<0.05$ were included in multivariate logistic regression model by a forward procedure. Then predictive nomogram was generated based on converting regression coefficient to a 0 - to 100-point scale proportionally. Predictive performance of model was measured by validation, discrimination and decision analysis[14]. Calibration curve was generated with bootstrap samples to reduce the overfit bias[15]. Hosmer-Lemeshow (HL) test implied good calibration when the test is insignificant. Discriminative performance was assessed by area under the receiver operating characteristic (ROC) curve. In addition, clinical usefulness of the nomogram is evaluated by decision curve analysis (DCA) by evaluating net benefits at different threshold probabilities. Survival analysis was also performed by univariate method with Kaplan-Meier analysis between low- risk and high-risk group according to the cut-off value of $50 \%$.

Statistical analysis was conducted using Statistical Package for the Social Sciences (SPSS) 24.0 and R software 3.5.0. All tests were considered significant when two-sided $P$ value was less than 0.05 .

\section{Results}

\section{Clinical Characteristics}

By March 24, 2020, a total of 1,540 patients with confirmed SARS-CoV-2 pneumonia from two Hospitals had been finally enrolled in the study. 1,068 patients were from Tongji Hospital; 472 from Wuhan Pulmonary Hospital. Of these patients, 268 patients failed to survive, with an in-hospital mortality around $16.1 \%$. 749 patients with SARS-CoV-2 pneumonia were included in the training set, 319 patients in the internal testing set and 472 patients in the external validation set. Social-demography, comorbidities, symptoms, vital signs, laboratory findings, and computed tomography results of different groups are described in Table 1. The results of training set are firstly described. The non-survival group had higher percentage of male patients than that of survival group ( 92 [66.7\%] vs $289[47.3 \%], P<0.001)$. The median age of non-survival patients from training set was older than that of survival patients $(68$ [IQR, $62-77]$ vs $58[$ [IQR,4567], $P<0.001)$. Majority of old individuals had comorbidities, such as hypertension, coronary heart disease, type 2 diabetes mellitus, underlying lung disease, and malignancy. The most common disease was hypertension (234[31.2\%]). Non-survival patients had significantly higher body temperature(T), respiratory rate(RR), and heart rate(HR) than those of survivors $\left(37.0^{\circ} \mathrm{C}\right.$ vs $36.8^{\circ} \mathrm{C}, P=0.009 ; 95 \mathrm{bpm}$ vs $89 \mathrm{bpm}, P=0.001 ; 24$ breaths/minute vs $20 \mathrm{breaths} / \mathrm{minute}, P<$ 0.001). As for the laboratory findings, non-survival patients had significantly higher levels of white blood cell count(WBC, 10.05 [6.27-13.34] vs 5.37 [4.06$\left.6.96] \times 10^{9} / \mathrm{L}, P<0.001\right)$, neutrophil count (N,8.88 [5.39-12.22] vs 3.44 [2.51-5.07] $\left.\times 10^{9} / \mathrm{L}, P<0.001\right)$, alanine aminotransferase (ALT, 29[18-41] vs 21[1436] $\mathrm{U} / \mathrm{L}, \mathrm{P}=0.001$ ), aspartate amino transferase (AST, 40[29-58] vs 25[19-36]U/L, $P<0.001$ ), total bilirubin (Tbil, 11.90[7.88-17.83] vs 8.60[6.30-11.50] $\mu \mathrm{mol} / \mathrm{L}, P<0.001$ ), blood urea nitrogen (BUN, 8.65[5.78-12.93] vs 4.30[3.30-5.40]mmol/L, $P<0.001$ ), serum creatinine (Scr, 86.00[62.00-125.25] vs 66.00[56.00-82.00] $\mathrm{mmol} / \mathrm{L}, P<0.001)$, serum potassium $\left(\mathrm{K}^{+}, 4.39[3.91-4.85] \mathrm{vs} 4.18[3.89-4.51] \mathrm{mmol} / \mathrm{L}, P=0.002\right), \mathrm{LDH}(512.00[398.50-665.75] \mathrm{vs}$ 247.00[198.00-316.00] U/L, $P<0.001$ ), high sensitive cardiac troponin I (hs-cTnl, 40.80[14.33-201.73] vs 5.80[2.30-17.10]pg/mL, $P<0.001)$, prothrombin time (15.30[14.28-17.22] vs 13.70[13.20-14.40]seconds, $P<0.001)$, d-dimer(3.74[1.59-21.00] vs 0.62[0.32-1.35] $\mathrm{gg} / \mathrm{mL}, P<0.001$, and PCT(0.30[0.13-0.90] vs $0.04[0.03-0.09] \mathrm{ng} / \mathrm{mL}, P<0.001)$ than those of survivors. While, the levels of lymphocyte count $\left(0.54[0.40-0.72]\right.$ vs $\left.1.09[0.78-1.54] \times 10^{9} / \mathrm{L}, P<0.001\right)$, platelet count $\left(159[120-221]\right.$ vs $\left.210[160-273] \times 10^{9} / \mathrm{L}, P<0.001\right)$, total serum protein (TP, 65.1([60.9-70.6] vs 69.4 [65.7-72.7] g/L, $\left.P<0.001\right)$, and serum albumin (ALB, 30.70[27.43-33.62] vs 36.50[32.90-40.30] g/L, $P<0.001$ ) in non-survival patients were all significantly decreased compared with those of survivors. However, no significant difference in red blood cell count, hemoglobin, and activated partial thromboplastin time (APTT) was found between survivors and non-survivors $(P>0.05)$. Almost all non-survival patients $(137 / 138[99.3 \%])$ presented infiltrated bilateral lesions involving 5 lung lobules in CT images. But in the survival group, 556/611(91.0\%) patients presented bilateral multi-lobular lesions. Only 56 patients (7.4\%) in training set showed with unilateral lesions in CT scan. Therefore, whether in survivors or non-survivors, bilateral multi-lobular lesions can be found in vast majority of patients with SARS-CoV-2 pneumonia. The similar results can be found in internal testing set and external validation set, respectively (Table 1). 
Table 1

Demographic, clinical, laboratory, and radiographic findings of patients on admission

\begin{tabular}{|c|c|c|c|c|c|c|c|c|c|}
\hline \multirow[t]{2}{*}{ Variables } & \multicolumn{3}{|l|}{ Training set } & \multicolumn{3}{|c|}{ Internal testing set } & \multicolumn{3}{|c|}{ External validation set } \\
\hline & $\begin{array}{l}\text { Survivors }(n= \\
611)\end{array}$ & $\begin{array}{l}\text { Non-survivors } \\
(n=138)\end{array}$ & $\begin{array}{l}P \\
\text { value }\end{array}$ & $\begin{array}{l}\text { Survivors }(n= \\
261)\end{array}$ & $\begin{array}{l}\text { Non-survivors } \\
(n=58)\end{array}$ & $\begin{array}{l}P \\
\text { value }\end{array}$ & $\begin{array}{l}\text { Survivors }(n= \\
420)\end{array}$ & $\begin{array}{l}\text { Non-survivors } \\
(n=52)\end{array}$ & $\begin{array}{l}P \\
\text { value }\end{array}$ \\
\hline \multicolumn{10}{|l|}{$\begin{array}{l}\text { Clinical } \\
\text { features }\end{array}$} \\
\hline Sex, male & $289(47.3 \%)$ & $92(66.7 \%)$ & $\begin{array}{l}< \\
0.001\end{array}$ & $122(46.7 \%)$ & $40(69.0 \%)$ & 0.002 & $202(48.1 \%)$ & $35(67.3 \%)$ & 0.009 \\
\hline Age, years & $\begin{array}{l}58.00(45.00- \\
67.00)\end{array}$ & $68(62-77)$ & $\begin{array}{l}< \\
0.001\end{array}$ & $\begin{array}{l}57.00(44.00- \\
66.00)\end{array}$ & $\begin{array}{l}68.50(58.50- \\
78.50)\end{array}$ & $\begin{array}{l}< \\
0.001\end{array}$ & $\begin{array}{l}57.00(48.00- \\
66.00)\end{array}$ & $\begin{array}{l}68.50(62.00- \\
74.25)\end{array}$ & $\begin{array}{l}<.001 \\
0.00\end{array}$ \\
\hline Hypertension & $174(28.5 \%)$ & $60(43.5 \%)$ & 0.001 & $73(28.0 \%)$ & $28(48.3 \%)$ & 0.003 & $136(32.4 \%)$ & $32(61.5 \%)$ & $\dot{0} 001$ \\
\hline $\begin{array}{l}\text { Coronary } \\
\text { heart } \\
\text { disease }\end{array}$ & $44(7.2 \%)$ & $16(11.6 \%)$ & 0.086 & $13(5.0 \%)$ & $11(19.0 \%)$ & 0.001 & $23(5.5 \%)$ & $8(15.4 \%)$ & 0.013 \\
\hline $\begin{array}{l}\text { Diabetes } \\
\text { mellitus }\end{array}$ & $71(11.6 \%)$ & $24(17.4 \%)$ & 0.066 & $41(15.7 \%)$ & $8(13.8 \%)$ & 0.714 & $58(13.8 \%)$ & $16(30.8 \%)$ & 0.002 \\
\hline $\begin{array}{l}\text { Underlying } \\
\text { lung } \\
\text { disease* }\end{array}$ & $31(5.1 \%)$ & $14(10.1 \%)$ & 0.024 & $4(1.5 \%)$ & $5(8.6 \%)$ & 0.012 & $29(6.9 \%)$ & $3(5.8 \%)$ & 1.000 \\
\hline Malignancy & $16(2.6 \%)$ & $8(5.8 \%)$ & 0.056 & $4(1.5 \%)$ & $3(5.2 \%)$ & 0.116 & $17(4.1 \%)$ & $1(1.9 \%)$ & 0.707 \\
\hline $\begin{array}{l}\text { Temperature, } \\
{ }^{\circ} \mathrm{C}\end{array}$ & $\begin{array}{l}36.80(36.50- \\
37.30)\end{array}$ & $\begin{array}{l}37.00(36.50- \\
37.79)\end{array}$ & 0.009 & $\begin{array}{l}36.80(36.40- \\
37.20)\end{array}$ & $\begin{array}{l}37.00(36.61- \\
37.50)\end{array}$ & 0.014 & $\begin{array}{l}36.6(36.4- \\
36.9)\end{array}$ & $\begin{array}{l}36.6(36.5- \\
36.9)\end{array}$ & 0.872 \\
\hline $\begin{array}{l}\text { Heart rate, } \\
\text { bpm }\end{array}$ & $\begin{array}{l}89.00(80.00- \\
100.00)\end{array}$ & $\begin{array}{l}94.80(81.00- \\
109.00)\end{array}$ & 0.001 & $\begin{array}{l}89.50(80.00- \\
103.00)\end{array}$ & $\begin{array}{l}\text { 99.50(86.23- } \\
109.25)\end{array}$ & 0.002 & $\begin{array}{l}86.50(79.25- \\
98.00)\end{array}$ & $\begin{array}{l}89.00(79.25- \\
107.75)\end{array}$ & 0.214 \\
\hline $\begin{array}{l}\text { Respiratory } \\
\text { rate, } \\
\text { breaths/min }\end{array}$ & $\begin{array}{l}20.00(20.00- \\
21.50)\end{array}$ & $\begin{array}{l}24.00(20.00- \\
32.25)\end{array}$ & $<001$ & $\begin{array}{l}20.00(20.00- \\
21.00)\end{array}$ & $\begin{array}{l}25.00(21.00- \\
34.00)\end{array}$ & $\begin{array}{l}< \\
0.001\end{array}$ & $\begin{array}{l}20.00(20.00- \\
22.00)\end{array}$ & $\begin{array}{l}23.50(20.00- \\
30.00)\end{array}$ & $\dot{0} .001$ \\
\hline \multicolumn{10}{|l|}{$\begin{array}{l}\text { Laboratory } \\
\text { findings }\end{array}$} \\
\hline $\begin{array}{l}\text { White blood } \\
\text { cell count, } \\
\times 10^{9} / \mathrm{L}\end{array}$ & $\begin{array}{l}5.37(4.06- \\
6.96)\end{array}$ & $\begin{array}{l}10.06(6.27- \\
13.34)\end{array}$ & $\begin{array}{l}< \\
0.001\end{array}$ & $\begin{array}{l}5.27(4.15- \\
6.81)\end{array}$ & $\begin{array}{l}9.62(5.73- \\
14.13)\end{array}$ & $\begin{array}{l}< \\
0.001\end{array}$ & $\begin{array}{l}5.74(4.36- \\
7.40)\end{array}$ & $\begin{array}{l}9.44(6.44- \\
13.07)\end{array}$ & $\dot{0} .001$ \\
\hline $\begin{array}{l}\text { Neutrophil } \\
\text { count, } \\
\times 10^{9} / \mathrm{L}\end{array}$ & $\begin{array}{l}3.44(2.51- \\
5.07)\end{array}$ & $\begin{array}{l}8.88(5.39- \\
12.22)\end{array}$ & $\dot{0} 001$ & $\begin{array}{l}3.40(2.43- \\
4.73)\end{array}$ & $\begin{array}{l}8.37(4.80- \\
12.75)\end{array}$ & $\begin{array}{l}<.001 \\
0.01\end{array}$ & $\begin{array}{l}3.75(2.66- \\
5.15)\end{array}$ & $\begin{array}{l}8.25(5.20- \\
12.00)\end{array}$ & $<$. \\
\hline $\begin{array}{l}\text { Lymphocyte } \\
\text { count, } \\
\times 10^{9} / \mathrm{L}\end{array}$ & $\begin{array}{l}1.09(0.78- \\
1.54)\end{array}$ & $\begin{array}{l}0.54(0.40- \\
0.72)\end{array}$ & $<001$ & $\begin{array}{l}1.15(0.83- \\
1.64)\end{array}$ & $\begin{array}{l}0.67(0.45- \\
0.89)\end{array}$ & $<0.001$ & $\begin{array}{l}1.21(0.74- \\
1.76)\end{array}$ & $\begin{array}{l}0.60(0.44- \\
0.89)\end{array}$ & $\begin{array}{l}< \\
0.001\end{array}$ \\
\hline $\begin{array}{l}\text { Red blood } \\
\text { cell count, } \\
\times 10^{9} / \mathrm{L}\end{array}$ & $\begin{array}{l}4.13(3.81- \\
4.56)\end{array}$ & $\begin{array}{l}4.12(3.72- \\
4.60)\end{array}$ & 0.782 & $\begin{array}{l}4.14(3.83- \\
4.56)\end{array}$ & $\begin{array}{l}4.05(3.37- \\
4.64)\end{array}$ & 0.151 & $\begin{array}{l}4.28(3.90- \\
4.66)\end{array}$ & $\begin{array}{l}4.05(3.19- \\
4.38)\end{array}$ & 0.001 \\
\hline $\begin{array}{l}\text { Hemoglobin, } \\
\mathrm{g} / \mathrm{L}\end{array}$ & $\begin{array}{l}127.00(117.00- \\
138.00)\end{array}$ & $\begin{array}{l}128.00(115.00- \\
143.00)\end{array}$ & 0.789 & $\begin{array}{l}128.00(117.00- \\
139.00)\end{array}$ & $\begin{array}{l}124.00(109.25- \\
146.50)\end{array}$ & 0.595 & $\begin{array}{l}129.0(117.25- \\
142.0)\end{array}$ & $\begin{array}{l}123.5(99.25- \\
134.75)\end{array}$ & 0.009 \\
\hline $\begin{array}{l}\text { Platelet } \\
\text { count, } \\
\times 10^{9} / \mathrm{L}\end{array}$ & $\begin{array}{l}210.00(160.00- \\
273.00)\end{array}$ & $\begin{array}{l}159.00(119.75- \\
221.25)\end{array}$ & $<001$ & $\begin{array}{l}211.00(165.00- \\
277.00)\end{array}$ & $\begin{array}{l}149.50(102.70- \\
224.00)\end{array}$ & $<.001$ & $\begin{array}{l}202.5(158.25- \\
261.0)\end{array}$ & $\begin{array}{l}151.0(95.25- \\
207.75)\end{array}$ & $\stackrel{<}{0.001}$ \\
\hline $\begin{array}{l}\text { APTT, } \\
\text { seconds }\end{array}$ & $\begin{array}{l}40.00(37.10- \\
43.42)\end{array}$ & $\begin{array}{l}41.04(36.40- \\
46.53)\end{array}$ & 0.057 & $\begin{array}{l}40.00(37.10- \\
43.00)\end{array}$ & $\begin{array}{l}41.16(37.20- \\
47.75)\end{array}$ & 0.090 & $\begin{array}{l}34.80(32.40- \\
37.60)\end{array}$ & $\begin{array}{l}37.90(33.17- \\
44.00)\end{array}$ & 0.001 \\
\hline PT, seconds & $\begin{array}{l}13.70(13.20- \\
14.40)\end{array}$ & $\begin{array}{l}15.30(14.28- \\
17.22)\end{array}$ & $\begin{array}{l}< \\
0.001\end{array}$ & $\begin{array}{l}13.80(13.30- \\
14.30)\end{array}$ & $\begin{array}{l}15.35(14.10- \\
17.15)\end{array}$ & $\begin{array}{l}<.001 \\
0.01\end{array}$ & $\begin{array}{l}12.90(12.30- \\
13.60)\end{array}$ & $\begin{array}{l}15.65(13.65- \\
17.40)\end{array}$ & $\dot{0} 001$ \\
\hline
\end{tabular}

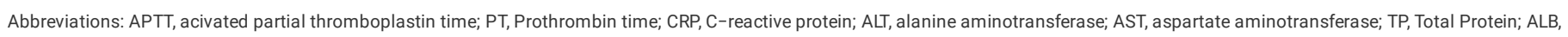

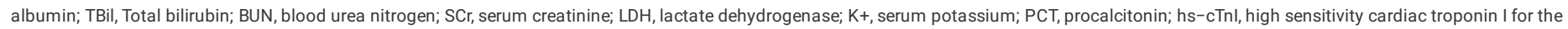
training and internal testing set; cTnl, cardiac troponin $\otimes$ for the external validation set

Data are characterized by $\mathrm{n}(\%)$ or median (IQR)

* underlying lung disease includes chronic obstructive pulmonary disease, asthma, bronchiectasis and tuberculosis etc 


\begin{tabular}{|c|c|c|c|c|c|c|c|c|c|}
\hline \multirow[t]{2}{*}{ Variables } & \multicolumn{3}{|l|}{ Training set } & \multicolumn{3}{|c|}{ Internal testing set } & \multicolumn{3}{|c|}{ External validation set } \\
\hline & $\begin{array}{l}\text { Survivors }(n= \\
611)\end{array}$ & $\begin{array}{l}\text { Non-survivors } \\
(n=138)\end{array}$ & $\begin{array}{l}P \\
\text { value }\end{array}$ & $\begin{array}{l}\text { Survivors }(n= \\
261)\end{array}$ & $\begin{array}{l}\text { Non-survivors } \\
(n=58)\end{array}$ & $\begin{array}{l}P \\
\text { value }\end{array}$ & $\begin{array}{l}\text { Survivors }(n= \\
420)\end{array}$ & $\begin{array}{l}\text { Non-survivors } \\
(n=52)\end{array}$ & $\begin{array}{l}P \\
\text { value }\end{array}$ \\
\hline $\begin{array}{l}\text { D-dimer, } \mu \mathrm{g} \\
/ \mathrm{mL}\end{array}$ & $\begin{array}{l}0.62(0.32- \\
1.35)\end{array}$ & $\begin{array}{l}3.74(1.59- \\
21.00)\end{array}$ & $\dot{<}_{0.001}$ & $\begin{array}{l}0.49(0.30- \\
1.01)\end{array}$ & $\begin{array}{l}4.30(1.20- \\
18.32)\end{array}$ & $\begin{array}{l}< \\
0.001\end{array}$ & $\begin{array}{l}0.35(0.16- \\
0.63)\end{array}$ & $\begin{array}{l}5.07(1.14- \\
15.18)\end{array}$ & $\stackrel{<}{0.001}$ \\
\hline CRP, mg/L & $\begin{array}{l}18.30(3.50- \\
60.60)\end{array}$ & $\begin{array}{l}102.65(58.57- \\
160.88)\end{array}$ & $\begin{array}{l}<.001 \\
0.00\end{array}$ & $\begin{array}{l}13.00(2.30- \\
48.80)\end{array}$ & $\begin{array}{l}99.48(66.43- \\
154.23)\end{array}$ & $\begin{array}{l}< \\
0.001\end{array}$ & $\begin{array}{l}12.64(1.14- \\
43.62)\end{array}$ & $\begin{array}{l}85.59(51.78- \\
102.50)\end{array}$ & $\begin{array}{l}<.001 \\
0.001\end{array}$ \\
\hline $\mathrm{ALT}, \mathrm{U} / \mathrm{L}$ & $\begin{array}{l}21.00(14.00- \\
36.00)\end{array}$ & $\begin{array}{l}29.00(18.00- \\
41.25)\end{array}$ & 0.001 & $\begin{array}{l}22.00(14.00- \\
36.00)\end{array}$ & $\begin{array}{l}29.00(17.00- \\
48.00)\end{array}$ & 0.002 & $\begin{array}{l}23.00(15.00- \\
37.00)\end{array}$ & $\begin{array}{l}30.50(19.00- \\
55.25)\end{array}$ & 0.017 \\
\hline AST, U/L & $\begin{array}{l}25.00(19.00- \\
36.00)\end{array}$ & $\begin{array}{l}39.50(28.75- \\
58.25)\end{array}$ & $<_{0.001}^{<}$ & $\begin{array}{l}24.00(19.00- \\
36.00)\end{array}$ & $\begin{array}{l}45.50(31.50- \\
71.00)\end{array}$ & $\begin{array}{l}< \\
0.001\end{array}$ & $\begin{array}{l}22.00(16.00- \\
33.00)\end{array}$ & $\begin{array}{l}42.50(27.00- \\
62.75)\end{array}$ & $\begin{array}{l}< \\
0.001\end{array}$ \\
\hline TP, g/L & $\begin{array}{l}69.41(65.66- \\
72.75)\end{array}$ & $\begin{array}{l}65.10(60.85- \\
70.63)\end{array}$ & $\dot{L}_{0.001}$ & $\begin{array}{l}69.90(66.20- \\
73.70)\end{array}$ & $\begin{array}{l}64.45(61.05- \\
71.00)\end{array}$ & $\begin{array}{l}< \\
0.001\end{array}$ & $\begin{array}{l}62.00(58.80- \\
66.18)\end{array}$ & $\begin{array}{l}60.85(56.25- \\
64.38)\end{array}$ & 0.026 \\
\hline ALB, g/L & $\begin{array}{l}36.50(32.90- \\
40.30)\end{array}$ & $\begin{array}{l}30.70(27.43- \\
33.62)\end{array}$ & $\hat{0}_{0.001}$ & $\begin{array}{l}37.50(33.90- \\
40.65)\end{array}$ & $\begin{array}{l}30.95(27.93- \\
34.30)\end{array}$ & $<_{0.001}$ & $\begin{array}{l}38.6(35.1- \\
42.1)\end{array}$ & $\begin{array}{l}33.5(30.85- \\
36.98)\end{array}$ & $\begin{array}{l}<.001 \\
0.001\end{array}$ \\
\hline TBil, $\mu \mathrm{mol} / \mathrm{L}$ & $\begin{array}{l}8.60(6.30- \\
11.50)\end{array}$ & $\begin{array}{l}11.90(7.88- \\
17.83)\end{array}$ & $\begin{array}{l}< \\
0.001\end{array}$ & $\begin{array}{l}8.40(6.25- \\
11.75)\end{array}$ & $\begin{array}{l}13.20(9.88- \\
19.05)\end{array}$ & $\begin{array}{l}< \\
0.001\end{array}$ & $\begin{array}{l}8.97(6.76- \\
11.92)\end{array}$ & $\begin{array}{l}13.60(9.73- \\
23.55)\end{array}$ & $<_{0.001}^{<}$ \\
\hline $\begin{array}{l}\mathrm{BUN}, \\
\mathrm{mmol} / \mathrm{L}\end{array}$ & $\begin{array}{l}4.30(3.30- \\
5.40)\end{array}$ & $\begin{array}{l}8.65(5.78- \\
12.93)\end{array}$ & $<_{0.001}^{<}$ & $\begin{array}{l}4.20(3.40- \\
5.40)\end{array}$ & $\begin{array}{l}9.29(5.68- \\
15.80)\end{array}$ & $\begin{array}{l}< \\
0.001\end{array}$ & $\begin{array}{l}4.91(3.80- \\
6.14)\end{array}$ & $\begin{array}{l}7.85(5.93- \\
12.77)\end{array}$ & $\begin{array}{l}< \\
0.001\end{array}$ \\
\hline $\mathrm{Scr}, \mu \mathrm{mol} / \mathrm{L}$ & $\begin{array}{l}66.00(56.00- \\
82.00)\end{array}$ & $\begin{array}{l}86.00(62.00- \\
125.25)\end{array}$ & $\dot{c}_{0.001}$ & $\begin{array}{l}67.00(56.00- \\
81.50)\end{array}$ & $\begin{array}{l}90.50(70.75- \\
133.50)\end{array}$ & $\begin{array}{l}< \\
0.001\end{array}$ & $\begin{array}{l}65.0(53.25- \\
78.75)\end{array}$ & $\begin{array}{l}82.5(66.25- \\
114.0)\end{array}$ & $\stackrel{<}{<.001}$ \\
\hline $\mathrm{LDH}, \mathrm{U} / \mathrm{L}$ & $\begin{array}{l}247.00(198.00- \\
316.00)\end{array}$ & $\begin{array}{l}512.00(398.50- \\
665.75)\end{array}$ & $\hat{0}_{0.001}$ & $\begin{array}{l}242.00(201.50- \\
310.50)\end{array}$ & $\begin{array}{l}514.00(398.75- \\
742.00)\end{array}$ & $\begin{array}{l}< \\
0.001\end{array}$ & $\begin{array}{l}218.00(162.00- \\
305.75)\end{array}$ & $\begin{array}{l}526.00(396.75- \\
719.60)\end{array}$ & $\begin{array}{l}<.001 \\
0.001\end{array}$ \\
\hline $\mathrm{K}^{+}, \mathrm{mmol} / \mathrm{L}$ & $\begin{array}{l}4.18(3.89- \\
4.51)\end{array}$ & $\begin{array}{l}4.39(3.91- \\
4.85)\end{array}$ & 0.002 & $\begin{array}{l}4.16(3.87- \\
4.50)\end{array}$ & $\begin{array}{l}4.38(4.01- \\
5.13)\end{array}$ & 0.005 & $\begin{array}{l}3.82(3.56- \\
4.17)\end{array}$ & $\begin{array}{l}3.82(3.54- \\
4.15)\end{array}$ & 0.825 \\
\hline PCT, ng/ml & $\begin{array}{l}0.04(0.03- \\
0.09)\end{array}$ & $\begin{array}{l}0.30(0.13- \\
0.90)\end{array}$ & $\hat{0}_{0.001}$ & $\begin{array}{l}0.04(0.03- \\
0.09)\end{array}$ & $\begin{array}{l}0.33(0.14- \\
1.03)\end{array}$ & $\begin{array}{l}< \\
0.001\end{array}$ & $\begin{array}{l}0.04(0.04- \\
0.04)\end{array}$ & $\begin{array}{l}0.28(0.10- \\
0.92)\end{array}$ & $\begin{array}{l}< \\
0.001\end{array}$ \\
\hline $\begin{array}{l}\text { hs-cTnl, } \\
\mathrm{pg} / \mathrm{mL} ; \text { cTnl, } \\
\mathrm{ng} / \mathrm{mL}\end{array}$ & $\begin{array}{l}5.80(2.30- \\
17.10)\end{array}$ & $\begin{array}{l}40.80(14.33- \\
201.73)\end{array}$ & $\stackrel{<}{0.001}^{-}$ & $\begin{array}{l}4.70(2.00- \\
11.34)\end{array}$ & $\begin{array}{l}61.45(11.00- \\
424.50)\end{array}$ & $\begin{array}{l}< \\
0.001\end{array}$ & $\begin{array}{l}0.02(0.01- \\
0.03)\end{array}$ & $\begin{array}{l}0.11(0.03- \\
0.28)\end{array}$ & $\stackrel{<}{0.001}$ \\
\hline $\begin{array}{l}\text { Computed } \\
\text { tomography }\end{array}$ & & & 0.001 & & & 0.217 & & & 0.023 \\
\hline $\begin{array}{l}\text { Unilateral } \\
\text { lesions }\end{array}$ & $55(9.0 \%)$ & $1(0.7 \%)$ & & 18(6.9\%) & $1(1.7 \%)$ & & $35(8.3 \%)$ & 0 & \\
\hline $\begin{array}{l}\text { Bilateral } \\
\text { lesions }\end{array}$ & $556(91 \%)$ & 137(99.3\%) & & $243(93.1 \%)$ & $57(98.3 \%)$ & & $385(91.7 \%)$ & $52(100 \%)$ & \\
\hline \multicolumn{10}{|c|}{$\begin{array}{l}\text { Abbreviations: APTT, acivated partial thromboplastin time; PT, Prothrombin time; CRP, C-reactive protein; ALT, alanine aminotransferase; AST, aspartate aminotransferase; TP, Total Protein; ALB, } \\
\text { albumin; TBil, Total bilirubin; BUN, blood urea nitrogen; SCr, serum creatinine; LDH, lactate dehydrogenase; Kt, serum potassium; PCT, procalcitonin; hs-cTnl, high sensitivity cardiac troponin I for the } \\
\text { training and internal testing set; CTnl, cardiac troponin } ₫ \text { for the external validation set. }\end{array}$} \\
\hline \multicolumn{10}{|c|}{ Data are characterized by n (\%) or median (IQR). } \\
\hline
\end{tabular}

\section{The Risk Factors Responsible For The Mortality}

In the training cohort, the univariate logistic regression analysis identified the following risk factors affecting in-hospital mortality of patients with SARS-CoV-2 pneumonia (Table 2): male, age $\geq 60$ years, $T \geq 38^{\circ} \mathrm{C}, \mathrm{HR} \geq 100 \mathrm{bpm}, \mathrm{RR} \geq 30 \mathrm{bpm}$, presence of comorbidities (hypertension and underlying lung disease), neutrophil count $\geq 7 \times 10^{9} / \mathrm{L}$, lymphocyte count $\leq 0.8 \times 10^{9} / \mathrm{L}$, platelet count $<100 \times 10^{9} / \mathrm{L}$, APTT $\geq 45$ seconds, PT $\geq 35$ seconds, d-dimer $\geq 1.5 \mu \mathrm{g} / \mathrm{mL}, \mathrm{CRP}$ $\geq 10 \mathrm{mg} / \mathrm{L}, A L T \geq 40 \mathrm{U} / \mathrm{L}$, AST $\geq 40 \mathrm{U} / \mathrm{L}, \mathrm{TP}<65 \mathrm{~g} / \mathrm{L}, \mathrm{ALB}<35 \mathrm{~g} / \mathrm{L}, \mathrm{Tbil} \geq 30 \mu \mathrm{mol} / \mathrm{L}, \mathrm{BUN} \geq 7 \mathrm{mmol} / \mathrm{L}, \mathrm{Scr} \geq 133 \mu \mathrm{mol} / \mathrm{L}, \mathrm{LDH} \geq 350 \mathrm{U} / \mathrm{L}, \mathrm{K}^{+} \geq 5.5 \mathrm{mmol} / \mathrm{L}$, $\mathrm{PCT} \geq 0.1 \mathrm{ng} / \mathrm{mL}$, and hs-cTnl $\geq 15.6 \mathrm{pg} / \mathrm{mL}$. To investigate roles of risk factors and their weights in the mortality of patients with SARS-CoV-2 pneumonia, we further performed a multivariable logistic regression analysis. Finally, eight risk factors among above were still significantly associated with in-hospital mortality of patients. As shown in Table 2, these important risk factors included that age $\geq 60$ years $(\mathrm{OR}=2.840 ; 95 \% \mathrm{Cl}, 1.467-5.495 ; P=0.002)$, respiratory rate $\geq 30$ breaths per minute $(\mathrm{OR}=3.308 ; 95 \% \mathrm{Cl}, 1.408-7.770 ; P=0.006)$, neutrophil count $\geq 7 \times 10^{9} / \mathrm{L}(\mathrm{OR}=3.084 ; 95 \% \mathrm{Cl}, 1.667-5.707 ; P<0.001)$, lymphocyte count $\leq 0.8 \times 10^{9} / \mathrm{L}(\mathrm{OR}=4.688 ; 95 \% \mathrm{Cl}, 2.500-8.791 ; P<0.001)$, d-dimer $\geq 1.5 \mu \mathrm{g} / \mathrm{mL}(\mathrm{OR}=2.159 ; 95 \% \mathrm{Cl}, 1.169-3.989 ; P=0.014), \mathrm{LDH} \geq$ $350 \mathrm{U} / \mathrm{L}(\mathrm{OR}=4.385 ; 95 \% \mathrm{Cl}, 2.299-8.362 ; P<0.001), \mathrm{PCT} \geq 0.1 \mathrm{ng} / \mathrm{mL}(\mathrm{OR}=4.972 ; 95 \% \mathrm{Cl}, 2.537-9.746 ; P<0.001)$, and presence of myocardial injury (hs-cTnl $\geq 15.6 \mathrm{pg} / \mathrm{mL}$ or $\mathrm{CTnl} \geq 0.1 \mathrm{ng} / \mathrm{mL} ; \mathrm{OR}=2.289 ; 95 \% \mathrm{Cl}, 1.260-4.160 ; P=0.007)$. 
Table 2

Univariate and multivariate logistic regression analysis in training set.

\begin{tabular}{|c|c|c|c|c|c|c|c|c|}
\hline \multirow[t]{2}{*}{ Variables } & \multicolumn{4}{|c|}{ Univariate analysis } & \multicolumn{4}{|c|}{ Multivariate analysis } \\
\hline & $\beta$ & OR & $\mathrm{Cl}$ & $P$ value & $\beta$ & OR & $\mathrm{Cl}$ & $P$ value \\
\hline Sex,male & 0.801 & 2.228 & $1.512-3.284$ & $<0.001$ & & & & \\
\hline Age $\geq 60$ years & 1.679 & 5.362 & $3.358-8.562$ & $<0.001$ & 1.044 & 2.840 & $1.467-5.495$ & 0.002 \\
\hline Temperature $\geq 38^{\circ} \mathrm{C}$ & 0.454 & 1.575 & $0.981-2.530$ & 0.060 & & & & \\
\hline Heart rate $\geq 100 \mathrm{bpm}$ & 0.678 & 1.969 & $1.345-2.883$ & $<0.001$ & & & & \\
\hline Respiratory rate $\geq 30 / \mathrm{min}$ & 2.380 & 10.806 & $6.210-18.803$ & $<0.001$ & 1.196 & 3.308 & $1.408-7.770$ & 0.006 \\
\hline Hypertension & 0.659 & 1.932 & $1.322-2.824$ & 0.001 & & & & \\
\hline Underlying lung disease* & 0.748 & 2.112 & $1.092-4.088$ & 0.026 & & & & \\
\hline Neutrophil count $\geq 7 \times 10^{9} / \mathrm{L}$ & 2.711 & 15.042 & $9.756-23.195$ & $<0.001$ & 1.126 & 3.084 & $1.667-5.707$ & $<0.001$ \\
\hline Lymphocyte count $\leq 0.8 \times 10^{9} / \mathrm{L}$ & 2.511 & 12.320 & $7.710-19.686$ & $<0.001$ & 1.545 & 4.688 & $2.500-8.791$ & $<0.001$ \\
\hline platelet count $<100 \times 10^{9} / \mathrm{L}$ & 1.729 & 5.636 & $3.056-10.397$ & $<0.001$ & & & & \\
\hline APTT $\geq 45$ seconds & 0.868 & 2.382 & $1.579-3.594$ & $<0.001$ & & & & \\
\hline $\mathrm{PT} \geq 15$ seconds & 2.186 & 8.898 & $5.903-13.412$ & $<0.001$ & & & & \\
\hline D-dimer $\geq 1.5 \mu \mathrm{g} / \mathrm{mL}$ & 2.427 & 11.326 & 7.327-17.509 & $<0.001$ & 0.770 & 2.159 & $1.169-3.989$ & 0.014 \\
\hline $\mathrm{CRP} \geq 10 \mathrm{mg} / \mathrm{L}$ & 2.642 & 14.038 & $6.096-32.326$ & $<0.001$ & & & & \\
\hline $\mathrm{ALT} \geq 40 \mathrm{U} / \mathrm{L}$ & 0.501 & 1.651 & $1.094-2.492$ & 0.017 & & & & \\
\hline $\mathrm{AST} \geq 40 \mathrm{U} / \mathrm{L}$ & 1.358 & 3.888 & $2.640-5.727$ & $<0.001$ & & & & \\
\hline $\mathrm{TP}<65 \mathrm{~g} / \mathrm{L}$ & 1.212 & 3.359 & $2.284-4.940$ & $<0.001$ & & & & \\
\hline ALB $<35 \mathrm{~g} / \mathrm{L}$ & 2.202 & 9.039 & $5.524-14.789$ & $<0.001$ & & & & \\
\hline TBil $\geq 30 \mathrm{umol} / \mathrm{L}$ & 2.865 & 17.550 & $4.828-63.823$ & $<0.001$ & & & & \\
\hline $\mathrm{BUN} \geq 7 \mathrm{mmol} / \mathrm{L}$ & 2.728 & 15.309 & $9.918-23.632$ & $<0.001$ & & & & \\
\hline Scr $\geq 133 \mathrm{umol} / \mathrm{L}$ & 2.138 & 8.482 & $4.711-15.270$ & $<0.001$ & & & & \\
\hline $\mathrm{LDH} \geq 350 \mathrm{U} / \mathrm{L}$ & 3.258 & 26.005 & $15.732-42.986$ & $<0.001$ & 1.478 & 4.385 & $2.299-8.362$ & $<0.001$ \\
\hline $\mathrm{K} \geq 5.5 \mathrm{mmol} / \mathrm{L}$ & 1.951 & 7.035 & $2.461-20.110$ & $<0.001$ & & & & \\
\hline PCT $\geq 0.1 \mathrm{ng} / \mathrm{ml}$ & 3.167 & 23.725 & $13.806-40.771$ & $<0.001$ & 1.202 & 4.972 & $2.537-9.746$ & $<0.001$ \\
\hline hs-cTnl $\geq 15.6 \mathrm{pg} / \mathrm{ml}$ & 2.036 & 7.659 & $5.033-11.654$ & $<0.001$ & 0.828 & 2.289 & $1.260-4.160$ & 0.007 \\
\hline
\end{tabular}

\section{Construction And Validation Of The Nomogram}

On the basis of eight variables from multivariable logistic regression analysis in training set, a predictive model was successfully constructed and visualized as a form of a nomogram plot to utilize in clinical practice (shown in Fig. 1). Calibration curves derived from training set showed a good fitting of the model with no statistical significance $(P=0.740)$ by Hosmer-Lemeshow test. The calibration curves indicated great consistence in training set, internal and external validating sets (Fig. 2). In Fig. 3, the ROC curve of the nomogram displayed more favorable discrimination than that of CURB-65 score in training set, with a larger area under curve (AUC $=0.956[0.941-0.971]$ vs $0.828[0.785-0.870], P<0.001)$. And AUC values by the predictive nomogram in both internal and external testing sets were superior than those calculated by CURB-65 score $(0.948$ vs $0.847, P<0.001$ and 0.949 vs $0.831, P<0.001)($ Fig. 3$)$. Each patient was divided into high-risk or low-risk group according to the cut-off value of $50 \%$ (death probability) predicted by nomogram. The three Kaplan-Meier survival curves derived from the data of training, internal, and external set were shown in Fig. 4. Kaplan-Meier survival curves all indicated that high-risk group had a much worse outcome than that of the low-risk group $(P<0.001)$. The Decision Curve Analysis (DCA) of predictive model showed a threshold probability of $5-$ 95\% (Fig. 5), in which the model had the ability to identify patients who might not survive superior to the "treat-all-patients" or "treat-none" schemes.

\section{Discussion}


Several previous studies have systematically summarized the features of SARS-Cov-2 pneumonia patients.[1, 5, 16, 17] Yang et al[9] described the characteristics of critically ill patients with SARS-CoV-2 pneumonia. At present, some studies have investigated the risk factors affecting mortality in SARSCoV-2 pneumonia[18], but papers constructing risk models to predict survival are very limited, which may be practical for doctors to make clinical decisions in the early stage.

In our training cohort, the mean age of non-survivors was older than survivors (68 years vs 58 years). Most of elders were accompanied by underlying diseases, including hypertension, coronary heart disease, lung underlying diseases, and diabetes mellitus, which were documented in other viral pneumonia studies, such as SARS-CoV-2 [9, 17], SARS [10], MERS [19]. As for vital signs, the levels of body temperature, heart rate, and respiratory rate were all higher in non-survivors than those of survivors. However, univariate and multivariate analyses between survivors and non-survivors showed that age ( $\geq 60$ years) and respiratory rate ( $\geq 30$ /minute) were significantly associated with hospital mortality of patients with SARS-CoV-2 pneumonia. Previous studies has shown that, the age deference between survivors and non-survivors was outstanding, such as SARS-CoV-2(mean (SD), 51.9 (12.9) vs 64.6 (11.2) )[9] and SARS (median (IQR), 55 (35-63) vs $70(56-76)$ )[10] in 28 days. Higher respiratory rate was a common sign of dyspnea for patients, with a probability of disease progress to acute respiratory distress syndrome (ARDS). Zhou Fei et al.[18] reported that respiratory rate in non-survival COVID-19 patients was much higher than that of survivors (34 [63\%] vs 22 [16\%], $P<0.001)$, and 50 of $54(93 \%)$ non-survival patients had presented as ARDS before death. Then, it's reasonable to consider that respiratory rate $(\geq 30$ /minute) was an independent risk factor for predicting mortality of patients with SARS-CoV-2 pneumonia.

Similar to a previous report[11], our univariate analysis had also shown a decrease in the count of lymphocyte and platelet, as well as an increase in LDH and d-dimer. The other mortality risk factors were increased neutrophil count, procalcitonin, BUN, hs-cTnl, AST and etc. As shown before, the lymphocyte count was much lower in non-survival patients. Previous study indicated that lymphocyte count $<0.8 \times 10^{9} / \mathrm{L}$ was an independent mortality risk factor for viral pneumonia[20]. In our study, reduced lymphocyte count was confirmed to be a vital and independent mortality associated risk factor by using multivariate logistic regression analysis $(\mathrm{OR}=4.853)$. It is presumed that lymphocytes are at an exhaustion state before the patients reached death. Nevertheless, WBC count of the non-survivors was significantly increased, especially the neutrophils in non-survivors were around 2.6-fold higher than survivors. Although many patients in our study had neutrophil count below $7 \times 10^{9} / \mathrm{L}$ and procalcitonin below $0.25 \mathrm{ng} / \mathrm{ml}$, most of non-survival patients showed the increased neutrophil count and procalcitonin. Elevated serum procalcitonin was regarded as one of most commonly used markers for bacterial infection[21]. Bacterial coinfection was known as a major cause of mortality H7N9 influenza pneumonia[22], and the most common pathogen detected by sputum or blood culture was Acinetobacter baumannii $[20,22]$. Bacterial co-infection not only indicated higher mortality but also longer hospital stay time and much more hospital care cost compared with non-bacterial co-infection[20]. Furthermore, elevated levels of WBC and neutrophils are thought to be a major contributory factor for disease progression.[23] Univariate and multivariate analysis indicated that increased neutrophil count ( $\left.\geq 7 \times 10^{9} / \mathrm{L}\right)$ was risk factor for mortality of patients. This result was documented in other studies showing that the neutrophil count was elevated in non-survivors of novel coronavirus infected pneumonia compared with survivors.[23] In our current study, procalcitonin was identified to be a very important mortality associated factor, because of much higher weight $(\mathrm{OR}=5.586)$ than other factors. Until now, there is rare report about procalcitonin contributing to the death of patients with SARS-CoV-2 pneumonia. Therefore, we concluded that lymphocyte count reduction and bacterial co-infection (increased neutrophil count and procalcitonin) are two leading risks for the mortality of patients with SARS-CoV-2 pneumonia.

In our study, we further evaluated the importance of LDH in SARS-CoV-2 pneumonia. The level of serum LDH was found to be significantly elevated in more than $70 \%$ of patients in our study. Moreover, LDH with a level $\geq 350 \mathrm{U} / \mathrm{L}$ was found to be associated with the risk of death in SARS-CoV-2 pneumonia patients by multivariate analysis. A previous study showed that LDH level was associated with the severity of various diseases including SARS[24]. It has been proved that the level of LDH was higher in ICU patients with SARS-CoV-2 pneumonia than that in non-ICU patients in Wuhan[25]. Other factors, such as the platelet count, APTT, PT, ALT, AST, CRP, TP, ALB, BUN, and Scr, were associated mortality of patients by univariate analysis, however, they were not identified as the risks of mortality by multivariate analysis in our study.

Cardiac complications (including heart failure, arrhythmia, or myocardial infarction) are common in patients with pneumonia, especially in critical illness [26]. Coronary heart disease was shown to be correlated with acute cardiac events and poor prognosis in influenza and other viral pneumonia[27, 28]. Chen $\mathrm{C}$ et al. showed that cTnI was increased in critically ill patients with COVID-19, which was an independent risk factor of clinical disease status[29]. In our study, hscTnl or cTnl on admission was found be increased in more than half of non-survival patients. Elevated hs-cTnl or cTnl level on admission indicated myocardial injury, which was confirmed to be significantly associated with mortality of patients. This result was consistent with a recently published study[18]. Therefore, presence of myocardial injury on admission is another important risk factor for predicting the mortality of patients with SARS-CoV-2 pneumonia.

Higher coagulation activity was found in most hospitalized pneumonia patients (almost 90\%), and its main marker was increased d-dimer concentration[30]. In this study, d-dimer greater than $1.5 \mu \mathrm{g} / \mathrm{mL}$ is associated with mortality of SARS-CoV-2 pneumonia, which was documented in a previous study[18]. The elevation of d-dimer might be due to hypercoagulable state of patients during SARS-CoV-2 infection. Pathologists confirmed that hyaline thrombi were found in some microvessels of three cases with COVID-19 by minimally invasive autopsies [31]. Furthermore, anticoagulant therapy was found to be correlated with better prognosis in severe ill COVID-19 patients with increased level of D-dimer[32]. In addition, blood-borne tissue factor, which was found to be expressed on the cell surfaces of alveolar macrophages, neutrophils, and endothelial cells during lung infection, could highly induce systemic coagulopathy[33]. Therefore, it may predispose to thrombosis in the pulmonary micovessels of patients with SARS-CoV-2 pneumonia, presenting as the significant elevation of d-dimer.

Currently, CT scan is considered to be an effective modality for clinical diagnosis for viral pneumonia at early periods.[34] From our CT images in SARS-CoV-2 pneumonia, we concluded that infiltrated lesions in five lung lobes were shown in the majority of the infected patients. Ground-glass opacities and consolidation are the main features of CT. These CT imaging manifestations may have some differences from that in SARS. In SARS, CT imaging normally presents in the lower lung lobes with multifocal airspace consolidation in a short period.[35] Therefore, CT has limited value in predicting death in the patients 
with SARS-CoV-2 pneumonia, mainly because most cases presented as mutiple lobular infiltration (usually 5 lobes) or diffuse lesions on CT images whether in survival or non-survival patients.

Although many predictive models are available to evaluate the prognosis of pneumonia, including IDSA/ATS minor criteria, CRB-65, CURB-65, Halm criteria, qSOFA, PSI, SCAP, SIRS-Score, SMART-COP, SOFA for community-acquired pneumonia (CAP)[36] and MuLBSTA score for viral infection [37], there is a lack of predictive tool especially for coronavirus pneumonia mortality. By multivariate logistic regression analysis, age $(\geq 60$ years), respiratory rate $(\geq 30$

breaths/minute), neutrophil count ( $\left.\geq 7 \times 10^{9} / \mathrm{L}\right), \mathrm{PCT}(\geq 0.1 \mathrm{ng} / \mathrm{mL})$, lymphocyte count $\left(\leq 0.8 \times 10^{9} / \mathrm{L}\right), \mathrm{d}$-dimer $(\geq 1.5 \mathrm{ug} / \mathrm{mL}), \mathrm{LDH}(\geq 350 \mathrm{U} / \mathrm{L})$, and presence of myocardial injury were identified to be eight independent risks for mortality prediction for SARS-CoV-2 pneumonia patients. Then, we employed these eight risk factors and formulated a reliable mortality risk predictive model to stratify SARS-CoV-2 pneumonia patients. This model was also validated by both internal and external validation set, and the visual expression of the model was displayed by a nomogram. The good predictive performance of the nomogram is suggested by calibration, discrimination, survival curve analysis, and DCA. Furthermore, this nomogram had a better predictive ability than that of CURB-65 score both in training and validation sets.

The research has several limitations. First, this retrospective design may cause a potential and inherent selection bias. Secondly, most patients have not been tested for the levels of cytokines and the counts of T cells subgroup. Thus, we failed to consider these factors in multiple imputation and further analysis to avoid great bias from real situation. Through a simple comparison between groups, we found some interesting differences. We are planning to explore their significance in subsequent clinical studies. Third, all the patients included in our study are Chinese. The clinical features of patients might be different in other countries or areas. Then, there may be some inherent biases by using this study format. Our results should be further validated by the multiple-center, prospective study.

\section{Conclusion}

In summary, via this two-center retrospective study of patients diagnosed as SARS-CoV-2 pneumonia in Wuhan, we found some important mortality-related risks, including age $\left(\geq 60\right.$ years), respiratory rate( $\geq 30$ breaths/minute), neutrophil count $\left(\geq 7 \times 10^{9} / \mathrm{L}\right), \mathrm{PCT}(\geq 0.1 \mathrm{ng} / \mathrm{mL})$, lymphocyte count $\left(\leq 0.8 \times 10^{9} / \mathrm{L}\right)$, d$\operatorname{dimer}(\geq 1.5 \mathrm{ug} / \mathrm{mL}), \mathrm{LDH}(\geq 350 \mathrm{U} / \mathrm{L})$, and presence of myocardial injury. We firstly establish a reliable predictive nomogram model, which can accurately stratify patients into risk categories and predict early mortality of patients with SARS-Cov-2 pneumonia. This nomogram can guide clinicians to improve their abilities to evaluate patient prognosis and make further reasonable decisions.

\section{Abbreviation List}

AUROC, area under the receiver operator characteristic curve

ARDS, acute respiratory distress syndrome

COVID-19, Coronavirus Disease 2019

$\mathrm{CT}$, computed tomography

ECMO, extracorporeal membrane oxygenation

ICU, intensive care unit

IMV, invasive mechanical ventilation

IQR, interquartile range

LDH, lactate dehydrogenase

NIV, non-invasive mechanical ventilation

OR, odd ratio

ROC, receiver operator characteristic curve

SARS-CoV-2, severe acute respiratory syndrome coronavirus 2

Tnl, troponin I

WHO, World Health Organization

\section{Declarations}

\section{Declarations}

Ethics approval and consent to participate: The Ethics Commission of Tongji Hospital and Wuhan Pulmonary Hospital have all approved this study. The informed consent was obtained from patients involved before enrolment when data were collected retrospectively. 
Consent for publication: Consent for publication was obtained from that patients and their families.

Availability of data and materials: The datasets used and analyzed during the current study are available from the corresponding author on reasonable request. The $\mathrm{R}$ code for this paper is available on request from corresponding author.

Competing interests: We declare no competing interests.

Funding: none.

Authors' contributions: Drs S. Wei had full access to all of the data in the study and take responsibility for the integrity of the data and the accuracy of the data analysis. *Drs, M. Luo, JL. Lu, and J. Liu contributed equally and shared first authorship. Concept and design: S. Wei, M. Luo, JL. Lu. Acquisition, analysis, or interpretation of data: M. Luo, JL. Lu, J. Liu. Drafting of the manuscript: M. Luo, JL. Lu, S. Wei, SL. Xia. Critical revision of the manuscript for important intellectual content: M. Luo, JL. Lu, S. Wei. Statistical analysis: M. Luo, JL. Lu. Administrative, technical, or material support: J. Liu, S. Wei. All authors read and approved the final manuscript.

Acknowledgements: We thank all patients and their families involved in the study.

\section{References}

1. Huang C, Wang Y, Li X, Ren L, Zhao J, Hu Y, Zhang L, Fan G, Xu J, Gu X, et al: Clinical features of patients infected with 2019 novel coronavirus in Wuhan, China. The Lancet 2020.

2. XW X, XX W, XG J, KJ X, LJ Y, CL M, SB L, HY W, HN SZ. G et al: Clinical findings in a group of patients infected with the 2019 novel coronavirus (SARSCov-2) outside of Wuhan, China: retrospective case series. BMJ. 2020;368:m606.

3. Silverstein WK, Stroud L, Cleghorn GE, Leis JA. First imported case of 2019 novel coronavirus in Canada, presenting as mild pneumonia. The Lancet. 2020;395(10225):734.

4. Holshue ML, DeBolt C, Lindquist S, Lofy KH, Wiesman J, Bruce H, Spitters C, Ericson K, Wilkerson S, Tural A, et al: First Case of 2019 Novel Coronavirus in the United States. New England Journal of Medicine 2020.

5. Zhu N, Zhang D, Wang W, Li X, Yang B, Song J, Zhao X, Huang B, Shi W, Lu R, et al: A Novel Coronavirus from Patients with Pneumonia in China, 2019. New England Journal of Medicine 2020.

6. YM FWSZBY, ZG CWW, ZW SYH, JH T, YY T. P et al: A new coronavirus associated with human respiratory disease in China. Nature 2020.

7. Wang C, Horby PW, Hayden FG, Gao GF. A novel coronavirus outbreak of global health concern. The Lancet 2020.

8. DS AZ. H, S P: Middle East respiratory syndrome. Lancet. 2015;386(9997):995-1007.

9. Yang X, Yu Y, Xu J, Shu H, Xia Ja, Liu H, Wu Y, Zhang L, Yu Z, Fang M, et al: Clinical course and outcomes of critically ill patients with SARS-CoV-2 pneumonia in Wuhan, China: a single-centered, retrospective, observational study. The Lancet Respiratory Medicine 2020.

10. RA F, SE L, AS DH, WJ D, AS S, TE S. S: Critically ill patients with severe acute respiratory syndrome. JAMA. 2003;290(3):367-73.

11. TW L, TK K, D T, KM AESLKS, SL KYCCFY. B et al: Acute respiratory distress syndrome in critically ill patients with severe acute respiratory syndrome. JAMA. 2003;290(3):374-80.

12. Gomersall CD, Joynt GM, Lam P, Li T, Yap F, Lam D, Buckley TA, Sung JJY, Hui DS, Antonio GE, et al. Short-term outcome of critically ill patients with severe acute respiratory syndrome. Intensive Care Med. 2004;30(3):381-7.

13. JA S, IR W, JB C, MG MSPR, K, AM W, JR. C: Multiple imputation for missing data in epidemiological and clinical research: potential and pitfalls. BMJ. 2009;338:b2393.

14. Steyerberg EW, Vergouwe Y. Towards better clinical prediction models: seven steps for development and an ABCD for validation. Eur Heart J. 2014;35(29):1925-31.

15. Sakamoto Y, Yamauchi Y, Yasunaga H, Takeshima H, Hasegawa W, Jo T, Sasabuchi Y, Matsui H, Fushimi K, Nagase T. Development of a nomogram for predicting in-hospital mortality of patients with exacerbation of chronic obstructive pulmonary disease. Int J Chron Obstruct Pulmon Dis. 2017;12:160511.

16. Chen N, Zhou M, Dong X, Qu J, Gong F, Han Y, Qiu Y, Wang J, Liu Y, Wei Y, et al: Epidemiological and clinical characteristics of 99 cases of 2019 novel coronavirus pneumonia in Wuhan, China: a descriptive study. The Lancet 2020.

17. D W, B H, C H, F Z, X L, J Z, B W, H X, Z C, Y X et al: Clinical Characteristics of 138 Hospitalized Patients With 2019 Novel Coronavirus-Infected Pneumonia in Wuhan, China. JAMA 2020.

18. Zhou F, Yu T, Du R, Fan G, Liu Y, Liu Z, Xiang J, Wang Y, Song B, Gu X, et al. Clinical course and risk factors for mortality of adult inpatients with COVID-19 in Wuhan, China: a retrospective cohort study. Lancet. 2020;395(10229):1054-62.

19. A A-O YMA, F A-H YM, K AASBASSAA. AK, A A et al: Critically III Patients With the Middle East Respiratory Syndrome: A Multicenter Retrospective Cohort Study. Critical care medicine. 2017;45(10):1683-95.

20. Guo L, Wei D, Zhang X, Wu Y, Li Q, Zhou M, Qu J. Clinical Features Predicting Mortality Risk in Patients With Viral Pneumonia: The MuLBSTA Score. Front Microbiol. 2019;10:2752.

21. Gendrel D, Bohuon C. Procalcitonin as a marker of bacterial infection. Pediatr Infect Dis J. 2000;19(8):679-87. quiz 688. 
22. Gao HN, Lu HZ, Cao B, Du B, Shang H, Gan JH, Lu SH, Yang YD, Fang Q, Shen YZ, et al. Clinical findings in 111 cases of influenza A (H7N9) virus infection. N Engl J Med. 2013;368(24):2277-85.

23. AM J, L P, RJ SYPG. H, T D, R M: Role of tissue protection in lethal respiratory viral-bacterial coinfection. Science. 2013;340(6137):1230-4.

24. Lew TW, Kwek TK, Tai D, Earnest A, Loo S, Singh K, Kwan KM, Chan Y, Yim CF, Bek SL, et al. Acute respiratory distress syndrome in critically ill patients with severe acute respiratory syndrome. JAMA. 2003;290(3):374-80.

25. WJ G, ZY N, WH YH, CQ L, JX O, DSC HLLHSCLL H et al: Clinical Characteristics of Coronavirus Disease 2019 in China. The New England journal of medicine 2020.

26. Marrie TJ, Shariatzadeh MR. Community-acquired pneumonia requiring admission to an intensive care unit: a descriptive study. Medicine. 2007;86(2):103-11.

27. Corrales-Medina VF, Musher DM, Shachkina S, Chirinos JA. Acute pneumonia and the cardiovascular system. Lancet. 2013;381(9865):496-505.

28. Udell JA, Zawi R, Bhatt DL, Keshtkar-Jahromi M, Gaughran F, Phrommintikul A, Ciszewski A, Vakili H, Hoffman EB, Farkouh ME, et al. Association between influenza vaccination and cardiovascular outcomes in high-risk patients: a meta-analysis. JAMA. 2013;310(16):1711-20.

29. X X, C Y, L Z JQ, S J, D H, B C, Z Z, W G, Z L et al: Imaging and clinical features of patients with 2019 novel coronavirus SARS-CoV-2. European journal of nuclear medicine and molecular imaging 2020.

30. Milbrandt EB, Reade MC, Lee M, Shook SL, Angus DC, Kong L, Carter M, Yealy DM, Kellum JA, Gen IMSI. Prevalence and significance of coagulation abnormalities in community-acquired pneumonia. Mol Med. 2009;15(11-12):438-45.

31. Yao XH, Li TY, He ZC, Ping YF, Liu HW, Yu SC, Mou HM, Wang LH, Zhang HR, Fu WJ, et al. [A pathological report of three COVID-19 cases by minimally invasive autopsies]. Zhonghua Bing Li Xue Za Zhi. 2020;49(0):E009.

32. Tang N, Bai H, Chen X, Gong J, Li D, Sun Z. Anticoagulant treatment is associated with decreased mortality in severe coronavirus disease 2019 patients with coagulopathy. J Thromb Haemost 2020.

33. Giesen PL, Rauch U, Bohrmann B, Kling D, Roque M, Fallon JT, Badimon JJ, Himber J, Riederer MA, Nemerson Y. Blood-borne tissue factor: another view of thrombosis. Proc Natl Acad Sci U S A. 1999;96(5):2311-5.

34. O HSXHNJYC A, J G, Y F, C Z: Radiological findings from 81 patients with COVID-19 pneumonia in Wuhan, China: a descriptive study. The Lancet Infectious diseases 2020.

35. GC O, PL K, NL M, WC Y, LJ Z, S JCHBL, KW N. T: Severe acute respiratory syndrome: temporal lung changes at thin-section CT in 30 patients. Radiology. 2004;230(3):836-44.

36. K H PAPC, H FSMK. H, M B, FM B, K R, U V et al: Sequential organ failure assessment score is an excellent operationalization of disease severity of adult patients with hospitalized community acquired pneumonia - results from the prospective observational PROGRESS study. Crit Care (London England). 2019;23(1):110.

37. M LGDWXZYWQL. Z, J Q: Clinical Features Predicting Mortality Risk in Patients With Viral Pneumonia: The MuLBSTA Score. Frontiers in microbiology. 2019;10:2752.

\section{Figures}


Age(years)

RR(breath/minute)

$\mathrm{N}\left(\times 10^{9} / \mathrm{L}\right)$

$\mathrm{L}\left(\times 10^{9} / \mathrm{L}\right)$

D-dimer $(\mu \mathrm{g} / \mathrm{mL})$

$\mathrm{LDH}(\mathrm{U} / \mathrm{L})$

$\operatorname{PCT}(\mathrm{ng} / \mathrm{mL})$

Myocardiac injury: hs-cTnl $15.6 \mathrm{pg} / \mathrm{mL}$ or
cTnl $\geq 0 . \operatorname{lng} / \mathrm{mL}$

Total Points

Death probability $\geq 60$

$$
<60
$$

$\geq 30$

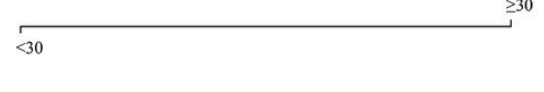

$<7$

$\geq 7$

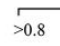

$\geq 1.5$

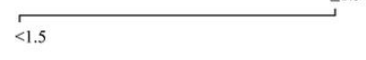

$<350$

$\geq 350$

$\geq 0.1$

$<0.1$

Presence

Absence

\begin{tabular}{llllllllllllll}
\hline 0 & 50 & 100 & 150 & 200 & 250 & 300 & 350 & 400 & 450 & 500 & 550 & 600 & 650
\end{tabular}

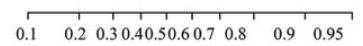

\section{Figure 1}

Nomogram model for early predicting in-hospital mortality in patients with SARS-CoV-2 pneumonia.

A

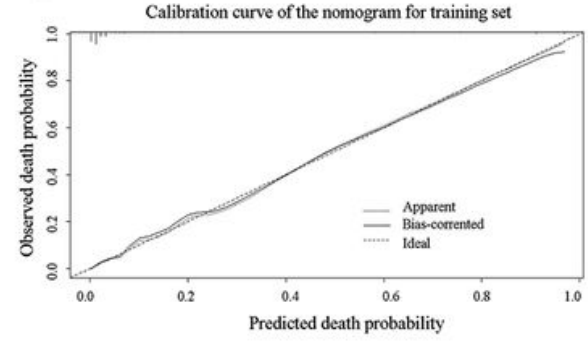

B

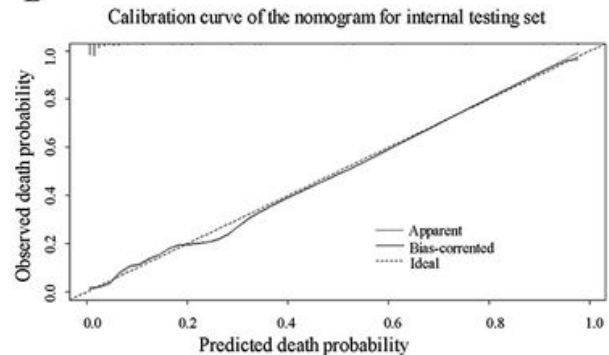

C Calbration curve of the nomogram for extemal validation set

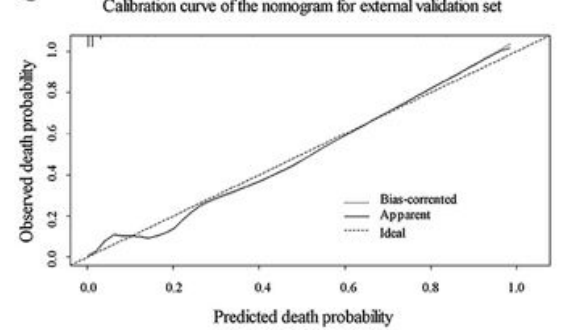

Figure 2

Calibration curves of the nomogram for predicting in-hospital mortality in patients with SARS-CoV-2 pneumonia. (A) Calibration curve of the nomogram for training set. (B) Calibration curve of the nomogram for internal testing set. (C) Calibration curve of the nomogram for external validation set. The $x$-axis represents the predicted mortality by nomogram. The y-axis represents the observed mortality. The diagonal dashed line represents for a perfect prediction by an ideal model. The solid line stands the performance of the nomogram, which has a good predictive ability. 
A

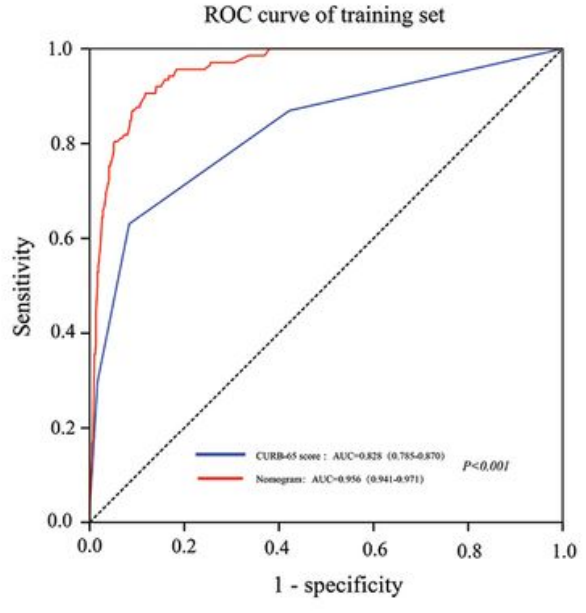

B

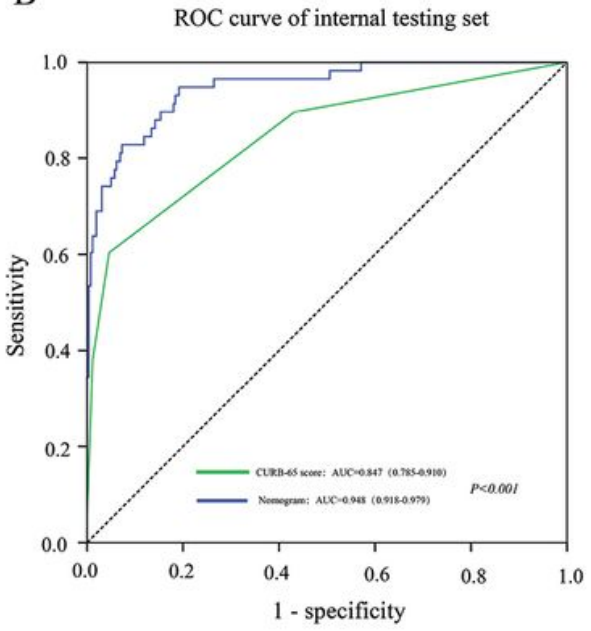

$\mathrm{C}$

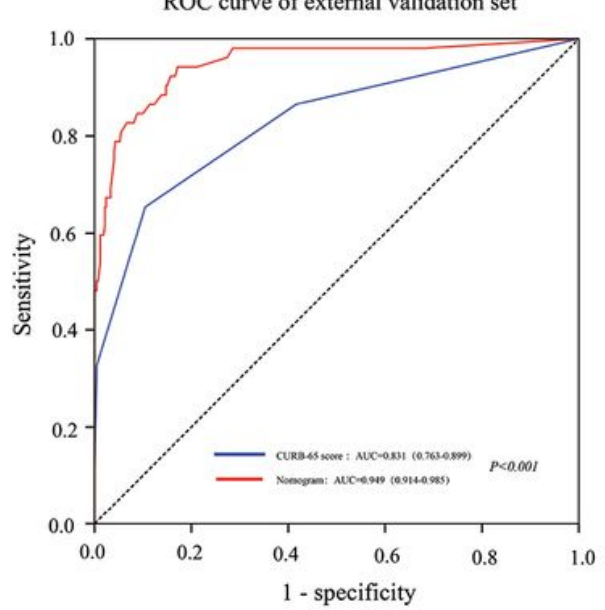

Figure 3

ROC curve analysis to early predict in-hospital mortality in patients with SARS-CoV-2 pneumonia. (A) ROC curve for the training set. (B) ROC curve for the internal testing set. (C) ROC curve for the external validation set. $A U C=a r e a$ under ROC curve; ROC= receiver operating characteristic. The three ROC curves derived by nomogram prediction all have better performance than those of CURB-65 $(\mathrm{P}<0.001)$.
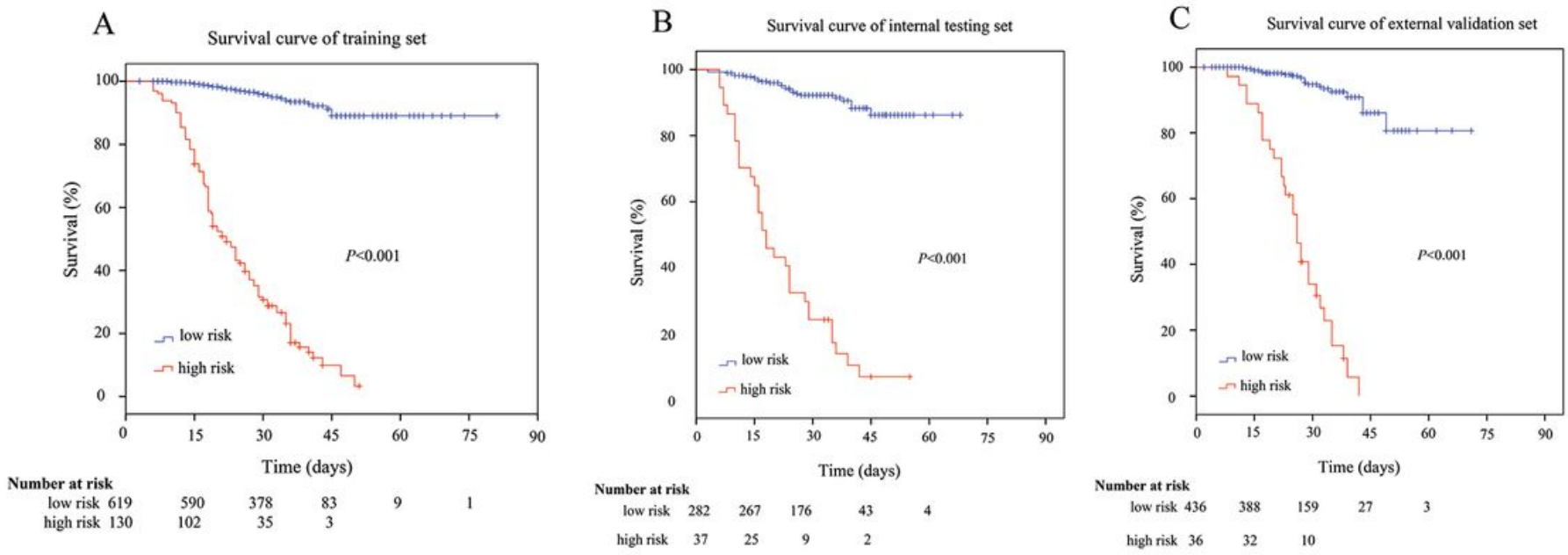

\section{Figure 4}

Kaplan-Meier curves of survival in hospitalized patients with SARS-CoV-2 pneumonia according to nomogram prediction $₫(A)$ training set $(n=749)$, (B) Internal testing set $(n=319)$, and $(C)$ External validation cohort $(n=472)$. Survival analysis was performed by univariate method with Kaplan-Meier analysis between low-risk and high-risk group according to the cut-off value of $50 \%$. 


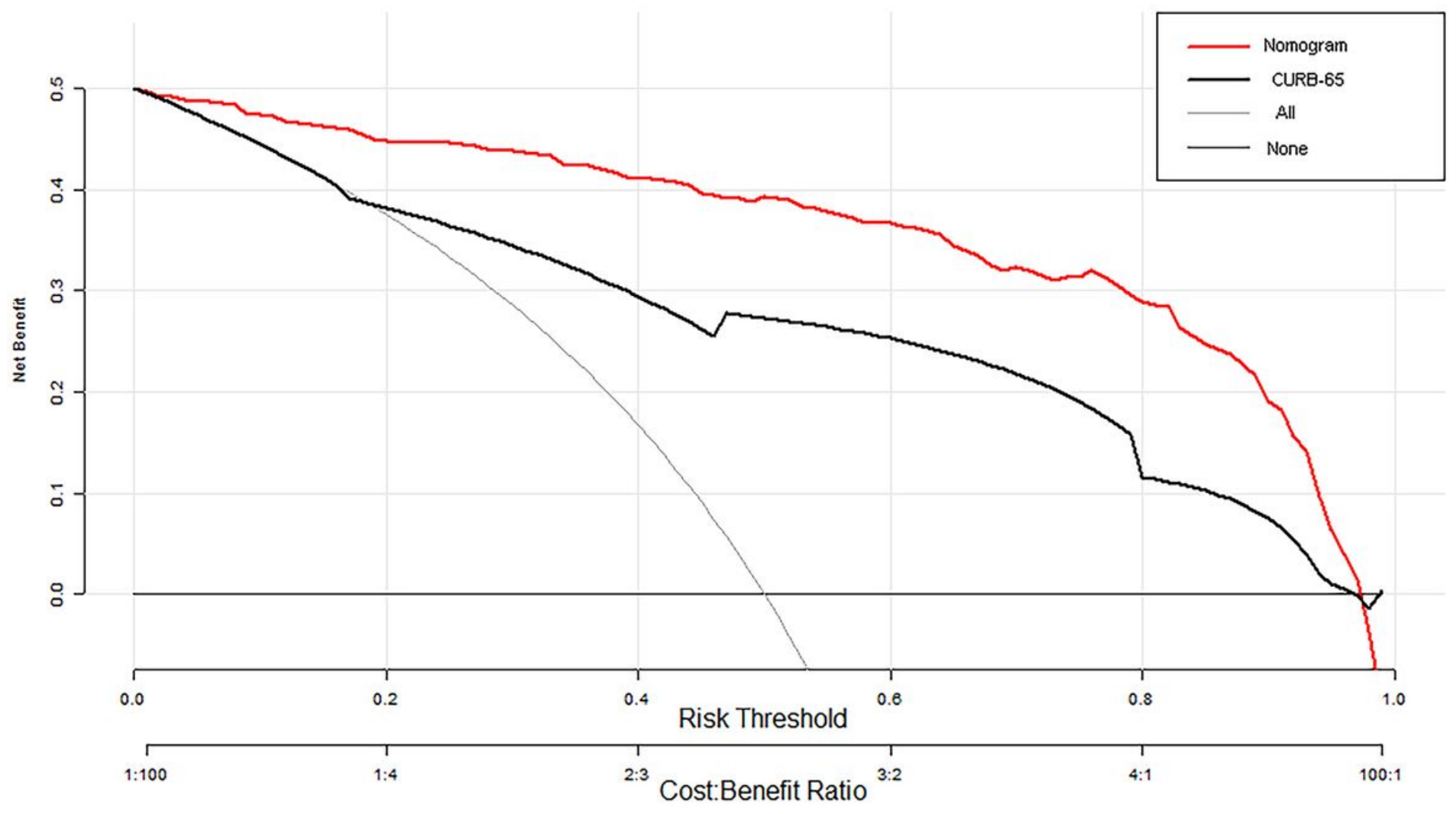

Figure 5

Decision curve analysis for early predicting in-hospital mortality in patients with SARS-CoV-2 pneumonia. The y-axis stands the net benefit. The red line stands the nomogram for predicting in-hospital mortality. The black and thick line stands the CURB-65 for predicting in-hospital mortality. The grey line displays the assumption that all patients have died. The fine and black line represents the assumption that no patients have died. The decision curve showed that predicting the death risk applying this nomogram would be better than that of CURB-65, having all patients or none patients treated by this nomogram with a range of the threshold probability between $>5 \%$ and $<95 \%$. 